\title{
Sex differences in predictors of illness intrusiveness 1 year after a cardiac event
}

\author{
Renée-Louise Franche ${ }^{\mathrm{a}, \mathrm{b}, \mathrm{c}, *}$, Susan Abbey ${ }^{\mathrm{b}, \mathrm{c}}$, Jane Irvine ${ }^{\mathrm{b}, \mathrm{d}}$, Zachary M. Shnek ${ }^{\mathrm{c}, \mathrm{e}}$, \\ Sherry L. Grace ${ }^{b}$, Gerald M. Devins ${ }^{b, c}$, Donna E. Stewart ${ }^{b, c}$
}

\begin{abstract}
Objective: This prospective study examined sex differences in illness intrusiveness and in baseline predictors of illness intrusiveness in cardiac patients' 1-year postcardiac event. Methods: Patients diagnosed with a myocardial infarction (MI) or unstable angina (UA) completed a baseline questionnaire during their hospitalization (139 men and 104 women). Eighty-one men and 67 women completed the follow-up questionnaire 1-year postcardiac event. The questionnaire included measures of illness intrusiveness in three life domains, depressive symptomatology, social support, medical information, risk factors for heart disease, and sociodemographic information. Results: A MANCOVA with the three Illness Intrusiveness subscales as dependent variables, sex as the betweengroup factor, and partnership status as a covariate found a sex effect for the Intimacy subscale, but not for the Instrumental and the Relationships/Personal Development subscales. Men reported more intrusiveness in the Intimacy domain of their life as compared to women. After controlling for sociodemographic factors and risk
\end{abstract}

factors for heart disease, hierarchical regressions indicated sex differences in baseline predictors of 1-year follow-up illness intrusiveness in the three life domains. For men, being satisfied with support from partner at baseline was associated with less intrusiveness in the Instrumental Life domain 1 year after the cardiac event. For women, surprisingly, more satisfaction with support from their partner at baseline was associated with more intrusiveness in their intimate lives 1 year after their cardiac event. In addition, emotional symptoms of depression at baseline were predictive of increased illness intrusiveness in Relationship/Personal Development and Intimacy domains for men and in Instrumental and Intimacy domains for women. Conclusion: Support from partner may have a differential impact for men and women after a cardiac event. Depressive symptomatology during initial hospitalization, particularly emotional symptoms, is a prominent indicator of illness intrusiveness for both sexes 1 year after the cardiac event.

\section{Introduction}

Quality of life issues following a cardiac event have been found to predict subsequent morbidity and mortality, independent of known prognostic factors [1]. Quality of life encompasses multiple aspects, ranging from strict consideration of physical symptoms to the inclusion of psychological states, as well as the degree of interference in a person's usual activities. It is the latter aspect of quality of life, namely illness intrusiveness, which is the focus of the current study.

Illness intrusiveness arises as a result of illness-induced lifestyle disruptions that interfere with involvement in valued activities $[2,3]$. It represents a fundamental aspect of the psychosocial impact of chronic medical conditions and of quality of life and is explained not only by the physical impact of the illness but also by ones' psychological and social resources. The construct of illness intrusiveness has been validated in studies involving individuals with rheumatoid arthritis, end stage renal disease (ESRD), multiple sclerosis [3-6], diabetes [7], laryngectomy [8], and 
breast cancer [9]. Differences in types of illness intrusiveness between patient groups have been documented, supporting the discriminant validity of the construct [2]. To date, illness intrusiveness, as measured by the Illness Intrusiveness Scale [2], has not been examined longitudinally in individuals following a cardiac event.

During the first 3 months following a traumatic event such as a cardiac event, one experiences decreased quality of life and increased psychological distress as part of an acute adjustment phase [10-12]. We have chosen to direct our attention to the longer term impact of a cardiac event on illness intrusiveness and therefore will review results of prospective studies only, pertaining to a period of at least 6 months duration following a cardiac event.

Prospective studies have examined predictors of various components of quality of life in individuals following a cardiac event. Poorer emotional quality of life at 6 months follow-up was predicted by poorer baseline emotional quality of life in patients with myocardial infarction (MI), and in patients with angina, by work disability, previous MI, smoker status, single status, and poorer baseline emotional quality of life [13]. In individuals with MIs, decreased social quality of life was predicted by baseline depressive and anxiety symptoms [14], smoking status, single status, having an MI prior to angina, and poorer emotional quality of life [13]. In individuals with angina, decreased social quality of life was predicted by poorer emotional quality of life [13]. In terms of constructs more closely related to illness intrusiveness, perceived decrease in leisure activities and subjective sense of "doing less" was predicted by baseline depressive and anxiety symptoms 1 year after having an MI [14]. Single status was not associated with poorer quality of life in patients with a history of ischemic heart disease 1 year after the cardiac event [11]. When evaluating the methodological quality of the above studies, it is important to note that in Mayou's (2000) [14] and Heller's (1997) [13] studies, level of physical symptomatology at follow-up was not controlled for, leaving open the possibility that follow-up level of distress was confounded with physical symptoms.

Sex differences in quality of life have been explored in only a few studies. One year after a cardiac event, women continued to report poorer quality of life in the areas of perceived general health [11] and psychological well-being as compared to men $[11,15]$. Compared to normal controls, men reported significantly poorer quality of sexual life 1 year after a cardiac event, while this was not the case for women despite similar baseline characteristics [11]. Women also reported experiencing more somatic symptoms as compared to normal controls 1 year after a cardiac event while men did not [11]. As well, 1-2 years after an MI, women reported more days of reduced activity due to an impaired state of health than men did [16].

Sex differences in illness intrusiveness in cardiac patients have not been examined previously. Given that heart disease is a chronic medical condition, with known sex differences in general quality of life, it is important to examine potential sex differences in illness intrusiveness.

The first objective of this study was to examine sex differences in illness intrusiveness, a crucial aspect of quality of life, 1 year after an MI or unstable angina (UA). The second objective was to determine if there are any sex differences in predictors of illness intrusiveness, with predictors being measured at time of initial diagnosis and illness intrusiveness measured 1 year after the cardiac event. We investigated the predictive power of depressive symptomatology and indices of social support measured at the time of initial diagnosis while controlling for sociodemographic factors, risk factors for heart disease, and physical symptoms.

\section{Material and methods}

\section{Procedure}

A research nurse screened the admission record daily of the Coronary Intensive Care Unit (CICU) for patients admitted with a diagnosis of MI or UA. Medical information was collected for all patients admitted to the CICU with MI or UA to enable later comparisons between participants and nonparticipants. The research nurse approached patients for participation in the study using a standardized text. They did not approach patients in the following circumstances: if a patient was in a lot of pain, was too emotionally upset, was on their way for a test or surgery, or was undergoing a line insertion procedure. In such circumstances, patients were approached the following day.

Participants were asked to complete a 20 -min questionnaire at the time of their admission to the CICU without assistance from family members. A similar questionnaire was completed at home 6 months and 1 year after their first admission. Only the 1-year follow-up data will be reported in this article.

\section{Participants}

\section{Eligibility criteria}

Patients in the CICU were eligible for participation if they met the following criteria:

admitting diagnosis is MI or UA

patient sufficiently alert and oriented to complete a questionnaire

patient not judged to be too ill

spoke and read English sufficiently to complete the questionnaire independently

patient not participating in a study, which conflicts with the current study

Of 573 potential participants, 373 patients admitted to the CICU fulfilled the eligibility criteria and were approached to 
participate in the study. Of those, $110(29 \%)$ refused to participate. Reasons for refusing to participate were the following: lack of interest (44\%), not feeling well enough $(34 \%)$, participating in another study $(1 \%)$, and other reasons (28\%). Twenty-one patients $(5.6 \%)$ agreed to participate but did not complete the questionnaire during their admission. There were no sex differences in reasons for refusals. At time of first admission, 139 men and 104 women completed the questionnaire for a participation rate of $65 \%$ of potential eligible participants.

\section{Instrumentation}

\section{Demographic information}

Basic demographic information was extracted from the medical chart by the research nurse and collected via selfreport in the questionnaire at baseline.

\section{Medical information}

Physical symptoms, risk factors for heart disease, medications used, and diagnostic tests received were assessed via self-report and by the research nurse from the medical chart.

\section{Satisfaction with social support}

Two questions assessed satisfaction with support from partner and from friends and family. For each item, five response categories were possible, ranging from "very unsatisfactory" to "very satisfactory."

\section{Beck Depression Inventory (BDI; [17])}

This 21-item measure of depressive symptomatology has been widely used in studies in the medically ill and in cardiac studies in particular. It has been shown by FrasureSmith et al. $[18,19]$ to be a stronger predictor of morbidity and mortality between 6 and 12 months post-MI than the diagnosis of major depression by structured interview. The instrument has demonstrated reliability and validity in the cardiac population. Scores range between 0 and 63. Two subscales, one pertaining to emotional symptomatology and the other to vegetative symptomatology, are differentiated in the BDI [20]. Given that medically ill individuals are likely to overreport somatic symptoms found in the vegetative subscale, the two subscales were analyzed separately in this study.

\section{Illness Instrusiveness Scale (IIS; [2])}

This 13-item scale measures illness-induced lifestyle disruptions interfering with involvement in valued activities.

An exploratory factor analysis conducted on 400 respondents from eight patient populations with distinct medical conditions shows the presence of three factors, which was cross validated with a second sample of 300 patients coming from seven of the eight patient populations [21]. The first factor, Relationships and Personal Development, includes six items: family relations, other social relations, self-expression, community involvement, and passive recreation. The second factor, instrumental life, includes four items: health, work, active recreation, and financial situation. The third factor, intimacy, includes two items: relationship with partner and sexual life. A diet item failed to meet criteria for inclusion in factor assignment. The psychometric properties of the scale include moderate to high levels of internal consistency reliability for subscales 1 (coefficient alpha ranges from .68 to .89), 2 (ranges from .66 to .89), and 3 (ranges from .58 to .90). Total scale internal consistency ranged from .82 to .94 . The scale has recently been incorporated into a number of studies of the medically ill $[2,8]$.

\section{Results}

\section{Preliminary analyses}

Differences among baseline participants, refusers, and ineligible patients

Differences in demographic characteristics were examined among baseline participants, ineligible patients, and patients refusing to participate. There were no differences in sex distribution, admitting diagnosis distribution (MI vs. UA), or proportions of married/common-law, separated/ divorced, and single individuals. Ineligible patients were significantly more likely to be widowed (Pearson $\chi^{2}=16.20$, $P=.013)$ and older than participants $(t=18.22, P<.001)$.

Sex differences in demographic and other descriptive variables were examined (see Table 1). There were no sex differences in diagnoses distribution. Significantly, more women were widowed than men in baseline participants (Pearson $\chi^{2}=11.22, P=.01$ ), nonparticipants (Pearson $\chi^{2}=13.85, P=.003$ ), and ineligible patients (Pearson $\left.\chi^{2}=17.32, P=.001\right)$. Women were significantly older than men in the participants $(t=2.278, P=.024)$ and ineligible groups $(t=3.768, P<.001)$.

Within the baseline participants group, there were no sex differences in education, diagnostic tests, medication, and time delay between first onset of symptoms and request for medical attention. Women reported significantly lower personal (Pearson $\chi^{2}=27.49, P<.001$ ) and family income (Pearson $\chi^{2}=15.00, P=.002$ ).

\section{Time 2-1-year follow-up: differences between participants and nonparticipants}

At the 1-year follow-up, 81 men (58\%) and $67(64 \%)$ women completed the questionnaire, representing $61 \%$ of the initial participant group. This participation rate is comparable to ones reported in previous studies using similar samples at 1-year follow-up- $-65 \%$ for MI patients [14] and $45 \%$ for MI and UA patients [22]. Differences in baseline measures between individuals who completed the 1-year follow-up and those who did not were examined (see Table 2). The 1-year follow-up participants had significantly 
Table 1

Baseline characteristics by sex of participants, refusers, and ineligible patients

\begin{tabular}{|c|c|c|c|c|c|c|}
\hline \multirow[b]{2}{*}{ Variable } & \multicolumn{2}{|l|}{ Participants } & \multicolumn{2}{|l|}{ Refusers } & \multicolumn{2}{|l|}{ Ineligible } \\
\hline & Males & Females & Males & Females & Males & Females \\
\hline Age & $60.8(10.85)$ & $64.1(11.39)$ & $63.6(10.73)$ & $65.4(13.33)$ & $66.2(11.42)$ & $72.2(9.53)$ \\
\hline \multicolumn{7}{|l|}{ Marital status } \\
\hline Married/common law & $110(82.1 \%)$ & $66(64.7 \%)$ & $40(83.3 \%)$ & $28(60.9 \%)$ & $60(76.0 \%)$ & $32(56.1 \%)$ \\
\hline Separated/divorced & $9(6.7 \%)$ & $12(11.8 \%)$ & $2(4.2 \%)$ & $3(6.5 \%)$ & $2(2.5 \%)$ & $1(1.8 \%)$ \\
\hline Single & $7(5.2 \%)$ & $6(5.9 \%)$ & $5(10.4 \%)$ & $2(4.3 \%)$ & $8(10.1 \%)$ & $1(1.8 \%)$ \\
\hline Widowed & $8(6.0 \%)$ & $18(17.6 \%)$ & $1(2.1 \%)$ & $13(28.3 \%)$ & $9(11.4 \%)$ & $23(40.3 \%)$ \\
\hline \multicolumn{7}{|l|}{ Diagnoses } \\
\hline MI & $48(34.8 \%)$ & $24(23.1 \%)$ & $15(30.0 \%)$ & $19(33.9 \%)$ & $36(37.1 \%)$ & $28(35.4 \%)$ \\
\hline UA & $90(65.2 \%)$ & $80(76.9 \%)$ & $35(70.0 \%)$ & $36(66.1 \%)$ & $61(62.9 \%)$ & $51(64.6 \%)$ \\
\hline \multicolumn{7}{|l|}{ Origin of transfers } \\
\hline Emergency & $52(37.4 \%)$ & $26(25.5 \%)$ & $22(42.3 \%)$ & $16(30.2 \%)$ & $32(33.0 \%)$ & $26(35.2 \%)$ \\
\hline Another ward/floor & $10(7.2 \%)$ & $14(13.7 \%)$ & $8(15.4 \%)$ & $13(24.5 \%)$ & $23(23.7 \%)$ & $18(24.3 \%)$ \\
\hline Another hospital & $77(55.4 \%)$ & $62(60.8 \%)$ & $22(42.3 \%)$ & $24(45.3 \%)$ & $42(43.3 \%)$ & $30(40.5 \%)$ \\
\hline \multicolumn{7}{|l|}{ Diagnostic tests in last month (not mutually exclusive) } \\
\hline EKG/ECG ( $n=91$ males and 71 females) & $78(85.7 \%)$ & $62(87.3 \%)$ & & & & \\
\hline Other heart tests ( $n=72$ males and 63 females) & $42(58.3 \%)$ & $33(52.4 \%)$ & & & & \\
\hline Diagnosed heart condition ( $n=77$ males and 60 females) & $51(66.2 \%)$ & $37(61.7 \%)$ & & & & \\
\hline \multicolumn{7}{|l|}{ Time from symptom onset to request for medical attention } \\
\hline Did not wait & $28(22.8 \%)$ & $26(28.9 \%)$ & & & & \\
\hline Waited $1 \mathrm{~h}$ or less & $28(22.8 \%)$ & $21(23.3 \%)$ & & & & \\
\hline Waited $1-6 h$ & $28(22.8 \%)$ & $17(18.9 \%)$ & & & & \\
\hline Waited over $6 \mathrm{~h}$ & $10(8.1 \%)$ & $5(5.6 \%)$ & & & & \\
\hline Waited days & $20(16.3 \%)$ & $13(14.4 \%)$ & & & & \\
\hline Waited weeks & $9(7.3 \%)$ & $8(8.9 \%)$ & & & & \\
\hline \multicolumn{7}{|l|}{ Education } \\
\hline Elementary & $17(13.1 \%)$ & $16(16.8 \%)$ & & & & \\
\hline High school & $57(43.8 \%)$ & $42(44.2 \%)$ & & & & \\
\hline College/university & $56(43.1 \%)$ & $37(39.0 \%)$ & & & & \\
\hline \multicolumn{7}{|l|}{ Personal income } \\
\hline Less than CAN $\$ 25,000$ & $31(25.2 \%)$ & $47(54.7 \%)$ & & & & \\
\hline CAN $\$ 25,000-50,000$ & $44(35.8 \%)$ & $30(34.9 \%)$ & & & & \\
\hline CAN $\$ 50,000-75,000$ & $23(18.7 \%)$ & $6(7.0 \%)$ & & & & \\
\hline over CAN\$75,000 & $25(20.3 \%)$ & $3(3.5 \%)$ & & & & \\
\hline \multicolumn{7}{|l|}{ Family income } \\
\hline Less than CAN $\$ 25,000$ & $13(11.4 \%)$ & $23(31.9 \%)$ & & & & \\
\hline CAN $\$ 25,000-50,000$ & $37(32.5 \%)$ & $25(34.7 \%)$ & & & & \\
\hline CAN $\$ 50,000-75,000$ & $29(25.4 \%)$ & $13(18.1 \%)$ & & & & \\
\hline over CAN\$75,000 & $35(30.7 \%)$ & $11(15.3 \%)$ & & & & \\
\hline
\end{tabular}

Data are reported with raw numbers and percentages in brackets. Percentages are calculated on available data and rounded to the nearest decimal point.

more baseline risk factors than nonparticipants $(t=4.105$, $P<.000)$. Total number of symptoms reported at admission was not significantly different between participants and nonparticipants; however, nonparticipants were significantly more likely to report fainting (likelihood ratio $=11.87$, $P=.007)$ and feeling faint in the last month (likelihood ratio $=4.007, P=.045$ ) than participants. Participants were more likely to report chest pain than nonparticipants (likelihood ratio $=7.266, P=.007$ ). Nonparticipants were also more likely to report that they were taking "heart medication" in the last month (likelihood ratio $=4.50, P=.034$ ), taking antacids or other pills for indigestion in the last month (likelihood ratio $=4.21, P=.040$ ), taking antidepressants (likelihood ratio $=5.36, P=.021$ ), and taking antidepressants in the last 5 years (likelihood ratio $=5.82, P=.016$ ). No significant differences were found in age, personal and family income, education, other symptoms, delay between first onset of symptoms and request for medical help, self-rated level of social support, and depressive symptomatology.

Sex differences were found in the 1-year follow-up participants, whereby women continued to report lower personal (Pearson $\chi^{2}=16.168, P=.001$ ) and family income (Pearson $\chi^{2}=9.667, P=.022$ ). Women were also more likely to be separated/divorced or widowed than men (Pearson $\chi^{2}=7.927, P=.048$ ). No sex differences were found in age, initial diagnosis, education level, diagnostic tests received, medication taken at admission, and time delay between first onset of symptoms and request for medical attention.

In view of the personal and family income differences between men and women in the initial participants group and the 1-year follow-up group, we examined correlations between these two indices of socioeconomic status at baseline with the three Illness Intrusiveness subscales. Correlations were nonsignificant and these variables were 
therefore not included as covariates in subsequent analyses. We also examined the correlations between the dichotomous variable of presence of a partner with the three Illness Intrusiveness subscales. We therefore created a dummy variable reflecting whether or not participants were married or living common-law versus those who were not (separated/divorced/single/widowed). There are limitations to this approach since being separated, divorced, single, or widowed may not necessarily translate into an absence of a partner. However, restricting the coding of partnered participants to the group of clearly and unambiguously partnered participants was the most conservative approach. A significant correlation was found only for the Intimacy scale (Spearman's $r=.33, P<.001$ ). Consequently, the variable of presence of a partner was included in subsequent analyses when appropriate.

In a previous study [23], quality of life was poorer in patients admitted for angina as compared to those admitted for an MI. No significant differences in scores of illness intrusiveness were found between these two groups when an ANOVA was conducted. Consequently, the two groups were collapsed.

\section{Sex differences in Illness Intrusiveness domains}

A MANCOVA, with sex as the between-group factor, with partnership status as a covariate, and the three Illness

Table 2

Baseline characteristics of participants and nonparticipants at 1-year follow-up

\begin{tabular}{lcc}
\hline Baseline variables & Participants & Nonparticipants \\
\hline No. of risk factors $(\max =10)$ & $4.58(1.93)$ & $3.48(2.41)$ \\
No. of symptoms at admission $(\max =24)$ & $6.80(4.32)$ & $7.13(5.08)$ \\
Satisfaction with social support of partner & $9.03(1.89)$ & $9.40(1.35)$ \\
Satisfaction with social support of others & $8.41(2.38)$ & $8.00(3.18)$ \\
Total BDI & $10.04(2.17)$ & $10.63(6.91)$ \\
BDI-psychological & $4.65(5.36)$ & $4.81(4.94)$ \\
BDI-vegetative & $3.33(2.17)$ & $3.87(2.58)$ \\
Age & $62.05(10.61)$ & $61.39(12.03)$ \\
Sex & & \\
Male & $81(54.7 \%)$ & $72(59.0 \%)$ \\
Female & $67(45.3 \%)$ & $50(41.0 \%)$ \\
Admitting diagnosis & & \\
MI & $39(26.5 \%)$ & $44(36.4 \%)$ \\
UA & $108(73.5 \%)$ & $77(63.6 \%)$ \\
Marital status & & \\
Married/common law & $111(76.0 \%)$ & $85(73.3 \%)$ \\
Separated/divorced & $13(8.9 \%)$ & $10(8.6 \%)$ \\
Single & $8(5.5 \%)$ & $5(4.3 \%)$ \\
Widowed & $14(9.6 \%)$ & $16(13.8 \%)$ \\
Origins of transfer & & \\
Emergency & $46(31.5 \%)$ & $42(34.4 \%)$ \\
Another ward/floor & $11(7.5 \%)$ & $16(13.1 \%)$ \\
Another hospital & $89(61.0 \%)$ & $64(52.5 \%)$ \\
\hline
\end{tabular}

Categorical data are reported with raw numbers and percentages in brackets. Continuous data are reported with means and standard deviations in brackets. Percentages are calculated on available data and rounded to the nearest decimal point.
Table 3

Illness intrusiveness scores for male participants, female participants, and total sample of participants

\begin{tabular}{llll}
\hline $\begin{array}{l}\text { Domain of } \\
\text { intrusiveness }\end{array}$ & Men & Women & All participants \\
\hline Total score & $33.61(14.71)$ & $35.20(17.42)$ & $34.30(15.90)$ \\
Relationships & & & \\
$\quad$ and Personal & & & \\
$\quad$ Development & $2.25(1.32)$ & $2.31(1.63)$ & $2.28(1.45)$ \\
Instrumental & $3.17(1.68)$ & $3.45(1.77)$ & $3.20(1.72)$ \\
Intimacy & $2.94(1.62)$ & $2.36(1.80)$ & $274(1.70)$ \\
\hline
\end{tabular}

Intrusiveness subscales scores as dependent variables, was conducted (see Table 3 for means and standard deviations). A multivariate effect for sex was significant $(F=3.42$, $P=.019$ ). Univariate tests for a sex effect, with partnership status as a covariate, were nonsignificant for the Relationship and Personal Development subscale and for the Instrumental Life subscale. For the Intimacy subscale, there was a significant sex effect, where men reported more intrusiveness in this domain as compared to women $(F=4.15$, $P=.044)^{1}$

\section{Regressions on illness intrusiveness for men and women}

In accordance with the recommendation to stratify sex in order to assess sex differences more accurately, we examined the potential predictors of illness intrusiveness separately for each sex. Hierarchical regressions were conducted for each sex to examine factors measured at admission, which were predictive of the degree of illness intrusiveness in the three life domains at the 1-year followup. Predictor variables were entered in three blocks. Age and education were entered in Block 1. Total number of risk factors at admission and total number of reported symptoms at the 1-year follow-up were entered in Block 2. Satisfaction with social support from partner at admission, satisfaction with social support from others at admission, emotional subscale of BDI at admission, and somatic subscale of BDI at admission were entered in Block 3 . Marital status was also included in Block 3 but for regressions on Relationships and Personal Development and Instrumental, domains only.

For the Relationships and Personal Development subscale, results for men indicate that only $20 \%$ of the variance at the 1 -year follow-up was explained by total number of symptoms reported in the last 6 months $(\beta=.244$, $F=6.56, P=.013$ ) and by emotional symptoms of depression as measured by the BDI Emotional subscale $(\beta=.366$, $F=6.56, P=.001$ ). For women, $26 \%$ of the variance at the 1-year follow-up was explained by total number of

\footnotetext{
${ }^{1}$ The same analyses were conducted with personal and/or family income as covariates, both for baseline and I-year income levels. Results of the analyses were similar.
} 
symptoms reported in the last 6 months $(\beta=.530, F=14.03$, $P=.001$ ).

For the Instrumental Life subscale, for men, 34\% of the variance at the 1-year follow-up was explained by total number of symptoms reported in the last 6 months ( $\beta=.326, F=15.82, P=.001$ ), vegetative symptoms of depression $(\beta=.290, F=13.08, P=.001)$, and satisfaction with social support from partner at baseline $(\beta=-.289$, $F=12.17, P=.001)$. For women, $58 \%$ of the variance at the 1-year follow-up was explained by age $(\beta=.091$, $F=4.30, P=.046$ ), total number of symptoms reported in the last 6 months $(\beta=.618, F=16.57, P=.001)$, and emotional symptoms of depression at baseline $(\beta=.367$, $F=17.81, P=.001$ ).

For the Intimacy subscale, in view of the fact that support from partner was a predictive variable, only partnered individuals were included in the analyses (68 men and 37 women). For men, only $11 \%$ of the variance at the 1 -year follow-up was explained by emotional symptoms of depression ( $\beta=349, F=8.32, P=.005$ ). For women, $74 \%$ of the variance at the 1-year follow-up was explained by total number of symptoms reported in the last 6 months $(\beta=.548$, $F=20.37, P=.001$ ), emotional symptoms of depression at baseline $(\beta=.691, F=34.37, P=.001)$, and social support from partner at baseline $(\beta=.279, F=28.95, P=.001)$. Surprisingly, it is increased social support from partner that is associated with increased intrusiveness in the Intimacy domain for women.

\section{Baseline sex differences in risk factors and psychosocial predictors of illness intrusiveness}

In order to explore sex differences at baseline in levels of social support from partner and from others, emotional and vegetative depressive symptoms, and number of physical symptoms, $t$-tests were conducted first with the initial participants group at baseline, and second with the remaining participants at the 1-year follow-up. Only the vegetative depressive symptoms were significantly higher in women than in men both in the initial participants group $(t=2.2016$, $P=.045$ ) and in the 1 -year follow-up participants group $(t=2.385, P=.018)$.

\section{Stability of the depressive symptomatology over 1 year}

The stability of total BDI scores, BDI Emotional subscale scores, and BDI Vegetative subscale scores was examined by conducting three separate repeated measures ANOVAs, using BDI scores at baseline and at follow-up as dependent variables. The BDI total scores were significantly higher at baseline than at the 1-year follow-up $(F=4.78$, $P<.05)$. There was a trend for the vegetative scores to be higher at baseline $(F=3.58, P=.061)$, and there were no significant differences between baseline and follow-up BDI psychological scores.

\section{Discussion}

One year after a cardiac event, men report more illness intrusiveness in their intimate life as compared to women, which is consistent with results of a previous study [11]. The overall absence of sex differences in the instrumental and Relationship/Personal Development Life domains stands in contrast to previous research in the general area of quality of life $[16,24,25]$. This may be due to the fact that many of the previous studies focused on physical status [11,24] and psychological well-being $[11,15,24]$ and did not measure illness intrusiveness.

In order to evaluate the clinical significance of the degree of total illness intrusiveness reported by patients 1 year after a cardiac event, we compared the total scores of illness intrusiveness of our study sample with those of 110 patients with rheumatoid arthritis, 101 patients with end stage renal disease (ESRD), and 94 patients with multiple sclerosis [2]. The cardiac patients in our study reported lower levels of illness intrusiveness $(M=34.30$, S.D. $=15.90)$ than patients with rheumatoid arthritis $(M=37.9$, S.D. $=16.87)$, ESRD $(M=38.8$, S.D. $=16.83)$, and multiple sclerosis $(M=42.6$, S.D. $=14.56)$.

There were sex differences in baseline predictors of illness intrusiveness 1 year postcardiac event and in the degree to which they explained variance in the three life domains examined. The amount of variance explained for the domain of Relationship and Personal Development remained modest for both genders. For men, it was predicted by physical symptoms occurring in the last 6 months and by baseline emotional depressive symptomatology. For women, it was predicted by symptoms in the last 6 months only. For the two other subscales, the amount of variance explained was consistently larger for women, particularly for the Intimacy domain of life (74\%) despite lower statistical power due to a low number of partnered women. Intrusiveness in the Instrumental Life domain was predicted for men by more symptoms in the last 6 months, increased vegetative symptoms of depression at baseline, and decreased satisfaction with support from partner at baseline. For women, it was predicted by older age, more symptoms in the last 6 months, and increased emotional symptoms of depression at baseline. For the Intimacy domain, while increased emotional symptoms of depression predicted only $11 \%$ of variance for men, more symptoms in the last 6 months, increased emotional symptoms of depression at baseline, and increased satisfaction with support from partner at baseline were predictive of increased intrusiveness in the intimate lives of women.

These results suggest that support from partner may have differential impact for men and women. For men, being satisfied with support from their partner at baseline is associated with less interference in the instrumental domain. For women, surprisingly, more satisfaction with their partner at baseline is associated with more interference in their intimate lives 1 year after their cardiac event. 
Previous research has provided only weak support for the relevance of social support in determining quality of life and morbidity in cardiac patients. When examining prediction of various indices of quality of life in patients 1 year after an MI or CABS, social support predicted only $0-10 \%$ of variance, while functional and psychological status at 3 months explained $35-56 \%$ of the variance for post-MI and $44-53 \%$ of variance for post-CABS patients [26]. Social support significantly explained $5 \%$ of the variance in quality of life of survivors of cardiac arrest with underlying coronary heart disease [27]. Other studies have not substantiated the role of baseline social quality of life as a predictor of future quality of life but found evidence for the importance of emotional quality of life as a predictor of the 6-month quality of life [13]. The weakness or absence of effect of social support in the above studies may be due to the fact that these studies examined general social support. The results of our study combined with the existing literature suggests that at least for men, it is support from a intimate person like a spouse, which is relevant to their recovery process, and not the presence of social support from more emotionally distant sources. For women, higher satisfaction with partner at baseline appears to bring on more interference in their intimate lives. One can only speculate about the mechanisms mediating such an association: It is possible that for women in more intimate and supportive relationships, their partners suffer more greatly from decreased ability to engage in various physical activities, including sexual ones, compared to the partners of men in more supportive relationships. This may bring women to report more intrusiveness in their intimate lives than men, following a cardiac event. Alternatively, women who report higher satisfaction with their partner's support may in fact become disillusioned as their partner may not live up to their expectations for support.

Results of our study support the role of depressive symptomatology, particularly emotional symptoms, as a prominent predictor of future illness intrusiveness in individuals with MI or UA. This is consistent with results of longitudinal studies addressing the prediction of quality of life 6 months or more after a cardiac event $[13,14]$. A recent longitudinal study of $288 \mathrm{MI}$ patients also found baseline levels of depression to be the strongest predictor of quality of life 4 months after a cardiac event [28]. In the latter study, the other significant predictors were severity of infarction, partner status, and state anxiety. Previous cross-sectional research has substantiated a relationship between illness intrusiveness and depression in adults with type 2 diabetes [7], individuals with rheumatoid arthritis [29,30], ERSD, and multiple sclerosis [30]. However, given the crosssectional nature of the above studies, a causal relationship between illness intrusiveness and depression could not be established. The results of our study suggest the role of depressive symptomatology as a contributing factor to later illness intrusiveness; however, it is likely that a bidirectional relationship exists between the two constructs, whereby higher illness intrusiveness would in turn lead to increased depressive symptomatology, mapping the well-known "depressive spiral" phenomenon.

Of note is the fact that the level of emotional depressive symptomatology appeared to be relatively stable over the first year following a cardiac event, which is consistent with results of a recent study [26]. The significant difference in levels of total depressive symptomatology seemed to be mostly attributable to an elevation in vegetative depressive symptoms at baseline as compared to the 1-year follow-up as no differences were found between baseline and followup levels of emotional depressive symptomatology. This pattern would be consistent with some of the reported symptoms being associated with the physical repercussions of having had a recent cardiac event on sleep, eating habits, etc. It suggests that the initial baseline level of emotional depressive symptomatology may not be simply in reaction to the cardiac event but be representative of a more stable mood state. The proposed stability of emotional depressive symptomatology would also give more support to the role of depressed mood as a predictor of illness intrusiveness as opposed to being a correlate of illness intrusiveness. Only a large longitudinal cohort study of individuals at risk of a cardiac event, where mood would be measured both before and after a cardiac event, would elucidate the question of mood stability around the time of a cardiac event.

The significance of the findings of the study for the healthcare practitioner are twofold. First, men appear to experience more difficulties in their intimate lives than women, following a cardiac event, and the healthcare practitioner should be proactive about inquiring about issues related to intimacy. Secondly, depressive symptomatology and particularly emotional symptoms present shortly after a cardiac event is an important predictor of certain aspects of patients' future quality of life. Healthcare practitioners should be vigilant to these early signs of depression and not minimize their importance, viewing these symptoms as only temporary - our results suggest that they reflect a stable and continuing pattern.

Methodological limitations of the study pertain to the selection bias and generalizability of results. At the 1-year follow-up, participants had a higher number of baseline risk factors than nonparticipants, which may have motivated their continued participation in the study. As well, at the 1-year follow-up, nonparticipants were more likely to have reported at baseline that they were taking medication than participants, suggesting that they may have been more vulnerable to future distress as compared to participants. Given the small number of widowed participants, sex differences in impact of widowhood on reported levels of social support could not be explored. Despite the fact that the 1year follow-up participation rate of $61 \%$ compared favorably to participation rates reported in other studies involving cardiac patients, it does limit the generalizability of results. Regarding the regression analyses relative to the Intimacy subscale, the small sample size, particularly for women, 
raises the possibility that these analyses were insufficiently powered. The latter analyses require replication in future studies and the small sample size in the current study limits the generalizability of results.

The current study points to the need to further explore the predictors of quality of life in men and women. In particular, understanding the factors impacting on illness intrusiveness in the intimate domains of their lives deserves further attention. The relationship between mood and illness intrusiveness deserves exploration with longitudinal designs, as it may be a critical component of our understanding of individuals' coping with the sequela of cardiac events.

\section{Acknowledgments}

We acknowledge the support of the Heart and Stroke Foundation of Canada for a research grant to investigate sex differences in patients following a cardiac event to Drs. Abbey, Stewart, Irvine, and Shnek. We wish to thank all the cardiac patients who generously participated in this study as well as the nurses and attending staff of the Toronto General Hospital Coronary Intensive Care Unit, University Health Network. This research was supported in part by the Canadian Institutes of Health Research through a Senior Investigator Award to G.M. Devins.

\section{References}

[1] Lim LL, Johnson NA, O'Connell RL, Heller RF. Quality of life and later adverse health outcomes in patients with suspected heart attack. Aust NZ J Publ Health 1998;22(5):540-6.

[2] Devins GM, Edworthy SM, Seland TP, Klein GM, Paul LC, Mandin $H$. Differences in illness intrusiveness across rheumatoid arthritis, end-stage renal disease and multiple sclerosis. $\mathbf{J}$ of Nerv Ment Dis 1993;181(6):377-81.

[3] Devins GM. Illness intrusiveness and the psychosocial impact of lifestyle disruptions in chronic life-threatening disease. Adv Renal Replace Ther 1994;1(3):251-63 (Review, 59 Refs).

[4] Devins GM, Armstrong SJ, Mandin H, Paul LC, Hons RB, Burgess ED, Taub K, Schorr S, Letourneau PK, Buckle S. Recurrent pain, illness intrusiveness and quality of life in end-stage renal disease. Pain 1990;42(3):279-85.

[5] Devins GM, Beanlands H, Mandin H, Paul LC. Psychosocial impact of illness intrusiveness moderated by self-concept and age in endstage renal disease. Health Psychol 1997;16(6):529-38.

[6] Devins GM, Mandin H, Hons RB, Burgess ED, Klassen J, Taub K, Schorr S, Letourneau PK, Buckle S. Illness intrusiveness and quality of life in end-stage renal disease: comparison and stability across treatment modalities. Health Psychol 1990;9(2):117-42.

[7] Talbot F, Nouwen A, Gingras J, Belanger A, Audet J. Relations of diabetes intrusiveness and personal control to symptoms of depression among adults with diabetes. Health Psychol 1999;18(5):537-42.

[8] Devins GM, Stam J, Koopmans JP. Psychosocial impact of laryngectomy mediated by perceived stigma and illness intrusiveness. Can J Psychiatry 1994;39(10):608-16.

[9] Bloom JR, Stewart SL, Johnston M, Banks P. Intrusiveness of illness and quality of life in young women with breast cancer. Psychoonkologia 1998;7(2):89-100 (Mar-Apr).

[10] Smith RT, Hamalainen H, Kallio V, Ronnemaa T, Lind J, Puukka P,
Arstila M, Kuttila K. Resource support and recovery after myocardial infarction or coronary artery bypass surgery: a prospectives study. J Rehabil Res Dev 1997;20:11-28.

[11] Westin L, Carlsson R, Erhardt L, Cantor-Graac E, McNeil T. Differences in quality of life in men and women with ischemic heart disease. A prospective controlled study. SCJ, Scand Cardiovasc J 1999;33(3): $160-5$.

[12] Westin L, Carlsson R, Israelsson B, Willenheimer R, Cline C, McNeil TF. Quality of life in patients with ischaemic heart disease: a prospective controlled study. J Intern Med 1997;242(3):239-47.

[13] Heller RF, Lim LL, Valenti L, Knapp I. Predictors of quality of life after hospital admission for heart attack or angina. Int J Cardiol 1997;59(2):161-6.

[14] Mayou RA, Gill D, Thompson DR, Day A, llicks N, Volmink J, Neil A. Depression and anxiety as predictors of outcome after myocardial infarction. Psychosom Med 2000;62(2):212-9.

[15] Stern MJ, Pascale L, Ackerman A. Life adjustment postmyocardial infarction. Arch Intern Med 1977;137:1680-5.

[16] Conn VS, Taylor SG, Abele PB. Myocardial infarction survivors: age and gender differences in physical health, psychosocial state and regimen adherence. J Adv Nurs 1991;16:1026-34.

[17] Beck AT, Ward CH, Mendelson M, Mock J, Erbaugh J. An inventory for measuring depression. Arch Gen Psychiatry 1961;4:561-71.

[18] Frasure-Smith N. In-hospital symptoms of psychological stress as predictors of long-term outcome after acute myocardial infarction in men. American Journal of Cardiology 1991;67:121-7.

[19] Frasure-Smith N, Lesperance F, Talajic M. Depression following myocardial infarction: impact on 6-month survival. J Am Assoc 1993; 270:1819-25.

[20] Minden SL, Orav J, Reich P. Depression in multiple sclerosis. Gen Hosp Psych 1987;9:426-34.

[21] Devins GM, Dion R, Pelletier L, Shapiro CM, Abbey S, Raiz LR, Yitzchak MB, McGowan P, Kutner NG, Benalands H, Edworthy SM. Structure of lifestyle disruptions in chronic disease: a confirmatory factor analysis of the illness intrusiveness rating scale. Med Care 2001;39(10):1097-104.

[22] Shnek Z, Irvine J, Stewart D, Abbey S. Psychological factors and depressive symptoms in ischemic heart disease. Health Psychol 2003;20(2):141-5.

[23] Heller RF, Lim LL, Valenti L, Knapp J. A randomized controlled trial of community based counselling among those discharged from hospital with ischemic heart disease. Aust N Z J Publ Health 1995; 25:362-4.

[24] Lavie CJ, Milani RV. Effects of cardiac rehabilitation and exercise training on exercise capacity, coronary risk factors, behavioral characteristics and quality of life in women. Am J Cardiol 1995;75:340-3.

[25] Loose MS, Fernhall B. Differences in quality of life among male and female cardiac rehabilitation participants. J Cardiopulm Rehabil 1995; 15:225-331.

[26] Hamalainen H, Smith R, Puukka P, Lind J, Kallio V, Kuttila K, Ronnemaa $T$. Social support and physical and psychological recovery one year after myocardial infarction or coronary artery bypass surgery. Scand J Publ Health 2000;28(1):62-70.

[27] Motzer SU, Stewart BJ. Sense of coherence as a predictor of quality of life in persons with coronary heart disease surviving cardiac arrest. Res Nurs Health 1996;19(4):287-98.

[28] Lane D, Carroll D, Ring CD, Beevers G, Lip GYH. Effects of depression and anxiety on mortality and quality-of-life 4 months after myocardial infarction. J Psychosom Res 2000;49:229-38.

[29] Devins GM, Edworthy SM, Guthrie NG, Martin L. Illness intrusiveness in rheumatoid arthritis: differential impact on depressive symptoms over the adult lifespan. J Rheumatol 1992;19(5):709-15.

[30] Devins GM, Edworthy SM, Paul LC, Mandin H, Seland TP, Klein G, Costello CG, Shapiro CM. Restless sleep, illness intrusiveness and depressive symptoms in three chronic illness conditions: rheumatoid arthritis, end stage renal disease and multiple sclerosis. J Psychosom Res 1993;37(2):163-70. 